\title{
Full Mouth Rehabilitation of Severely Worn Dentition by an Interdisciplinary Approach
}

\author{
Seema Sathe ${ }^{1}$, Deepika Rathi², Anjali Borle ${ }^{3}$ \\ ${ }^{1}$ Department of Prosthodontics, SPDC, Sawangi, Wardha, Maharashtra, India. ${ }^{2}$ Department of Prosthodontics, SPDC, \\ Sawangi, Wardha, Maharashtra, India. ${ }^{3}$ Department of Prosthodontics, SPDC, Sawangi, Wardha, Maharashtra, India.
}

\section{INTRODUCTION}

Dental aesthetics play an important role as it positively accesses the self-confidence, social life, and overall well-being of adults. ${ }^{1}$ Dental carelessness in terms of daily oral hygiene practices, access to oral health resource, dietary habits of the patient and attitude toward oral health are associated with poor oral hygiene status, caries experience, and periodontal disease. ${ }^{2}$ Severely debilitated oral conditions which involve the maximum number of teeth, demand for more extensive and multidisciplinary treatment. ${ }^{3}$ Tooth wear can result from abrasion, attrition and erosion. Excessive occlusal wear is a combination of various processes and it rarely acts alone. ${ }^{4}$ Proper diagnosis and treatment planning is needed for rehabilitating such case. Attention must be placed on parafunctional habits like bruxism, restoration and maintenances of a healthy dentition. ${ }^{5}$ Aesthetic and functional rehabilitation of the worn-out dentition is always a significant clinical challenge. However, the rehabilitation of the severely worn dentition becomes more challenging when insufficient space for restoration is present. 6

This case report describes full mouth rehabilitation treatment of a patient of worn out dentition that presented with severe wear, who was clinically monitored to evaluate the adaptation to the removable occlusal splint during 2 and 4 weeks as trial period after raising vertical height to avoid further complications of treatment. Full mouth restoration of the severely worn teeth represents a significant clinical challenge to a Prosthodontist. Tooth wear has intrigued restorative dentists due to its multifactorial aetiology. Loss of tooth structure may occur through non-carious processes like abrasion, attrition, erosion and possibly abfractions, acting alone or in combination. This case report describes a 44-year-old male, who had unilateral headache from last two and half years. There was an aesthetically and functionally compromised tooth due to collapsed bite.

\section{PRESENTATION OF CASE}

A 44 year old male patient reported to the Department of Prosthodontics, Sharad Pawar Dental College \& Hospital, with a chief complaint of severely worn out teeth and unilateral headache since last two and half years. Extra oral examination was normal (Fig. 1). Intraoral examination revealed generalized attrition with a sharp enamel wear and severely collapsed bite due to formation of occlusal facet on posteriors. Severe discrepancy was noted between vertical dimension at rest and occlusion which was about $5 \mathrm{~mm}$. Accordingly diagnostic impression and mounting was made to diagnose case and finalize the sequence of treatment. (Fig. 2). Full mouth restoration was panned for this case, after completion of advised endodontic phase. Restorations planned were all ceramic crowns with incisors, canine, and premolars of maxillary and mandibular arch and all metal veneers of maxillary and mandibular posteriors.

\author{
Corresponding Author: \\ Dr. Seema Sathe, \\ Department of Prosthodontics, \\ SPDC, Sawangi (M), Wardha-442001, \\ Maharashtra, India. \\ E-mail: seemasathe2011@gmail.com
}

DOI: $10.14260 /$ jemds/2019/807

Financial or Other Competing Interests: None.

How to Cite This Article:

Sathe S, Rathi D, Borle A. Full mouth rehabilitation of severely worn dentition by an interdisciplinary approach. J. Evolution Med. Dent. Sci. 2019;8(49):3727-3729, DOI: 10.14260/jemds/2019/807

Submission 17-09-2019,

Peer Review 16-11-2019,

Acceptance 22-11-2019,

Published 09-12-2019. 
The maxillary and mandibular diagnostic impressions were made with irreversible hydrocolloid impression material and diagnostic cast was poured. Facebow records were made and transferred on semi adjustable (Hanau $\mathrm{H} 2$ ) articulator. Mounting of maxillary cast was done. (Fig. 3). A deprogramming device, Lucia jig was formulated with pattern resin to utilize the $2 \mathrm{~mm}$ of freeway space available and interocclusal record was made by using bite registration material and mandibular diagnostic cast was mounted. Soft splint was fabricated on duplicated cast and patient was asked to wear it for 2 weeks after which it is replaced by acrylic hard splint of proposed vertical for a duration of 4 weeks (Fig. 4). In the interim period, diagnostic mockup and waxup was carried out for pre aesthetic evaluation (Fig. 5). Putty index of finalized diagnostic waxup was made. Tooth preparations were carried out for maxillary and mandibular arch (Fig. 6) followed by provisionalization. Provisionalization was done using eugenol free cement for duration of 1 month (Fig. 7). Mutually protected occlusion was developed in this patient. Patient was recalled at regular intervals for evaluation of aesthetic and functions.. Accordingly tooth preparations were finished, followed by gingival retraction and final impressions with poly vinyl siloxane impression material along with face bow record and transfer. Final restorations were fabricated and bisque trial of prosthesis was done to check aesthetics and marginal adaptations. After altering all necessary corrections finally prosthesis was cemented in sequential manner according to hobo's technique ie:- posterior first followed by anteriors by using resin modified GIC (Fig. 8).
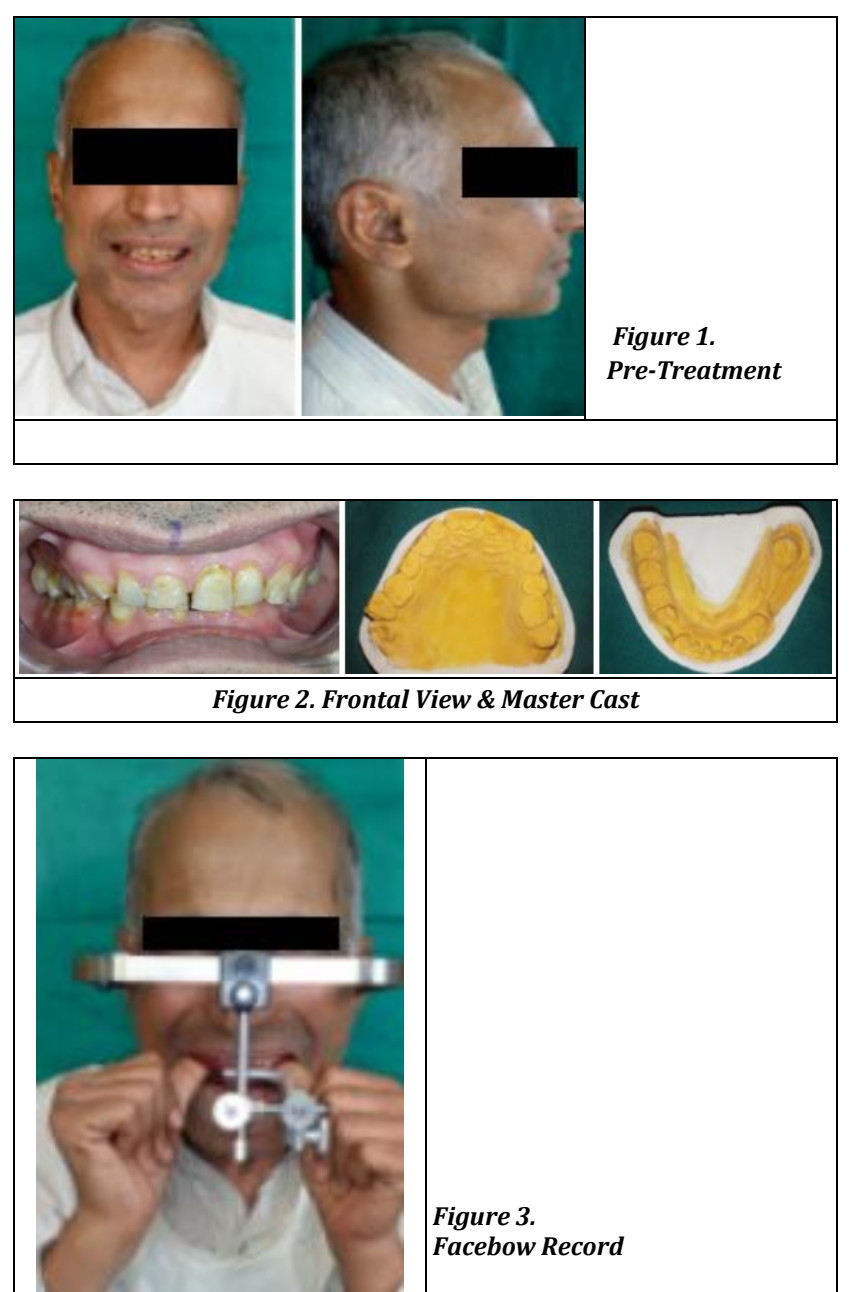

Figure 3.

Facebow Record
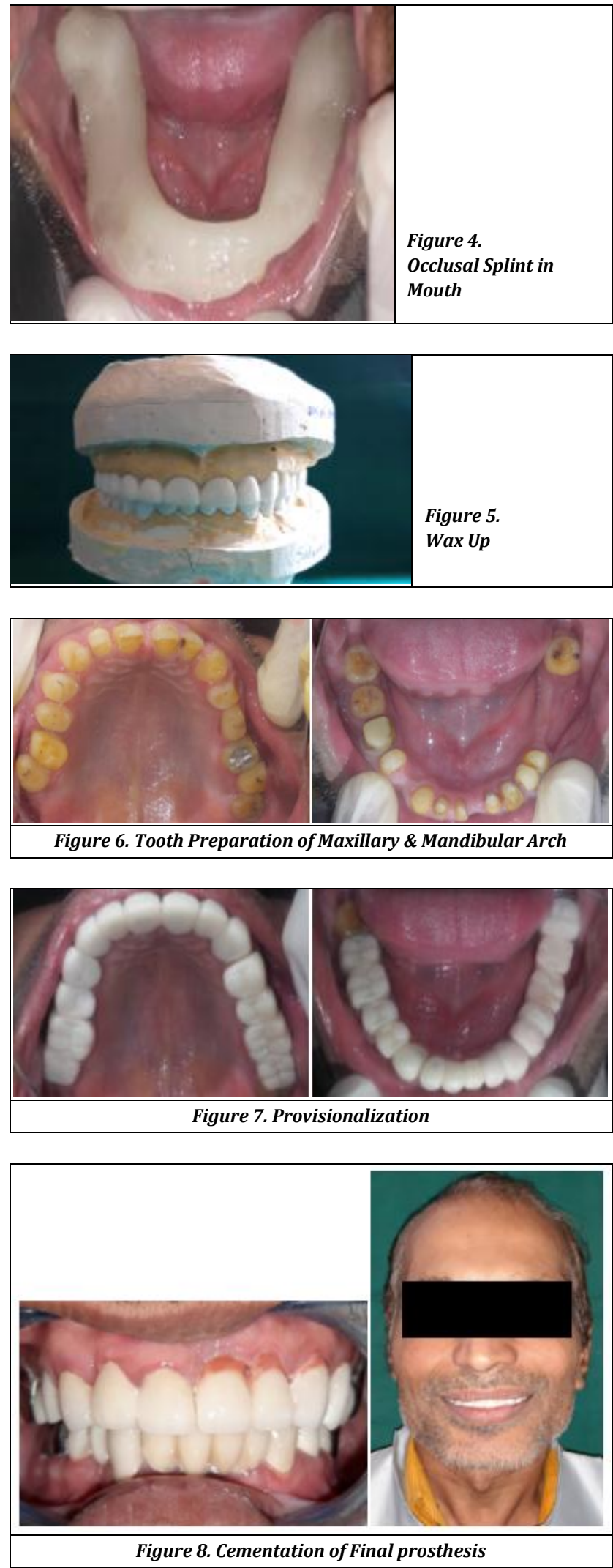

\section{DISCUSSION}

Full mouth restoration in severely attrited teeth is considered as one of the most challenging tasks for a dentist. Full mouth reconstructions involving full arch preparations, impressions, 
provisional restorations and master casts are regarded as simultaneous reconstructions. A variety of theories \& concepts may be used in simultaneous system to obtain complete arch dies and mounted casts.

In 1984, Turner classified the treatment of a severely worn dentition by the amount of the loss of VDO and available space to restore. His classification and conventional treatment, which includes raising VDO with multiple crown-lengthening procedures, have been widely used up to present. The wearing time of overlay splint and the provisional crown is varying in the literature. The trial period of reversible and conservative overlay prostheses is between 3 weeks to 5 months, and that of intensive fixed provisional prostheses is 2 to 6 months. In this case, the patient was carefully monitored for 1 month to evaluate the adaptation to the occlusal splints (Soft \& Hard splints were given). Also, the patient's adaptation to the provisional restoration was monitored for 1 month. ${ }^{7-12}$

As already discussed; there are many theories and concepts of Full mouth rehabilitation. In this case, we chose Hobo's technique for FMR. The rationale was to ensure that the occlusion which developed eventually resulted into predictable disocclusion of the posterior teeth during various eccentric movements. It is necessary to point at this stage that in most of the cases of generalized attrition, this is perhaps the most important thing to achieve.

In Hobo \& Takayama technique, pre- determined values are used for calculations and the operator can choose the type of occlusion, required for the case. The values are available for mutually protective occlusion, Group function \& balanced occlusion. The authors chose mutually protected occlusion in this case.

\section{CONCLUSIONS}

Full mouth rehabilitation is a type of restoration which specifically requires a team approach or multidisciplinary approach for a successful planning. A detailed case history and use of appropriate diagnostic tools in assessing the case yield satisfactory results. Management of severely worn dentition requires a precise protocol. Excess increase of VDO will worsen the condition. Tolerance of the patient to newly restored VDO is the chief priority in treatment. Full mouth rehabilitation restores the function, aesthetics and health of the dentition. In this case, successful full mouth rehabilitation with all ceramic zirconia crowns in incisors, canines and premolars of maxillary and mandibular arch and all metal restorations with posteriors of maxilla and mandible was accomplished. Patient was comfortable and satisfied in terms of function and aesthetics.

\section{REFERENCES}

[1] Klages U, Bruckner A, Zentner A. Dental aesthetics, self-awareness and oral health-related quality of life in young adults. Eur J Orthod 2004;26(5):507-14.

[2] Thomson WM, Locker D. Dental neglect and dental health among 26-year-olds in the Dunedin Multidisciplinary Health and Development Study. Community Dent Oral Epidemiol 2000;28(6):414-8.

[3] Klages U, Bruckner A, Guld Y, et al. Dental esthetics, orthodontic treatment and oral-health attitudes in young adults. Am J Orthod Dentofacial Orthop 2005;128(4):4429.

[4] Zinzala B, Javiya P, Sethuraman R, et al. Functional and esthetical full mouth rehabilitation of a patient with severely worn dentition: a clinical report. International Journal of Oral Health and Medical Research 2017;4(5):42-5

[5] Neff P. Trauma from occlusion. Restorative concerns. Dent Clin North Am 1995;39(2):335-54.

[6] Kalra A, Sandhu HS, Sahoo NK, et al. Full mouth rehabilitation using twin-stage technique. International Journal of Oral Health Sciences 2019;9(1):40-4.

[7] Ganddini MR, Al-Mardini M, Graser GN, et al. Maxillary and mandibular overlay removable partial dentures for the restoration of worn teeth. J Prosthet Dent 2004;91(3):210-4.

[8] Turner KA, Missirlian DM. Restoration of the extremely worn dentition. J Prosthet Dent 1984;52(4):467-74.

[9] Jahangiri L, Jang S. Onlay partial denture technique for assessment of adequate occlusal vertical dimension: a clinical report. J Prosthet Dent 2002;87(1):1-4.

[10] Hemmings KW, Darbar UR, Vaughan S. Tooth wear treated with direct composite restorations at an increased vertical dimension: results at 30 months. J Prosthet Dent 2000;83(3):287-93.

[11] Sato S, Hotta TH, Pedrazzi V. Removable occlusal overlay splint in the management of tooth wear: a clinical report. J Prosthet Dent 2000;83(4):392-5.

[12] Yunus N, Abdullah H, Hanapiah F. The use of implants in the occlusal rehabilitation of a partially edentulous patient: a clinical report. J Prosthet Dent 2001;85(6):5403. 